\title{
Germanica
}

\section{Nahrung und Gesellschaft - gemeinsames Essen in literarischen Darstellungen der DDR von Birk Meinhardt, Jochen Schmidt und Lutz Seiler}

Food and Society - Eating in Company in Literary Depictions of the GDR by Birk Meinhardt, Jochen Schmidt and Lutz Seiler

Alimentation et société - Manger ensemble dans les représentations littéraires de la RDA par Birk Meinhardt, Jochen Schmidt et Lutz Seiler

\section{Sandra Vlasta}

\section{OpenEdition}

\section{Journals}

Édition électronique

URL : http://journals.openedition.org/germanica/3041

DOI : 10.4000/germanica.3041

ISSN : 2107-0784

\section{Éditeur}

Université de Lille

\section{Édition imprimée}

Date de publication : 30 décembre 2015

Pagination : 99-114

ISBN : 9782913857360

ISSN : 0984-2632

\section{Référence électronique}

Sandra Vlasta, « Nahrung und Gesellschaft - gemeinsames Essen in literarischen Darstellungen der DDR von Birk Meinhardt, Jochen Schmidt und Lutz Seiler », Germanica [Online], 57 | 2015, Online erschienen am: 30 Dezember 2017, abgerufen am 06 Oktober 2020. URL : http://

journals.openedition.org/germanica/3041 ; DOI : https://doi.org/10.4000/germanica.3041 


\title{
Nahrung und Gesellschaft - gemeinsames Essen in literarischen Darstellungen der DDR von Birk Meinhardt, Jochen Schmidt und Lutz Seiler
}

\author{
Sandra VLASTA \\ Johannes Gutenberg - Universität Mainz.
}

\section{Einleitung}

Seit dem Ende der DDR sind einige literarische Verarbeitungen dieses Staates, vor allem seiner letzten Jahre, erschienen; viele dieser auch als ,Wenderomane" bezeichneten Bücher hatten großen Erfolg und wurden mit wichtigen Preisen ausgezeichnet ${ }^{1}$. So erhielt Lutz Seiler für Kruso (2014) den Deutschen Buchpreis 2014, Birk Meinhardts Brüder und Schwestern (2013) war für den Preis der Leipziger Buchmesse 2013 nominiert. In Zeiten des abnehmenden Lichts (2011) von Eugen Ruge wurde ebenfalls mit dem Deutschen Buchpreis (2011) sowie dem Aspekte Literaturpreis ausgezeichnet. Lutz Tellkamps Roman Der

1. - Für einen aktuellen Überblick über die Diskussion des Begriffs Wenderoman/literatur und seiner Alternativen siehe Sonja, Kersten: „Mauerfall-, Post-DDR, Vereinigungs-, Nachwende- oder doch Wendeliteratur?“, in: literaturkritik.de, 2015, 10. 
Turm (2008) schließlich erhielt neben einigen anderen Auszeichnungen gleichfalls den Deutschen Buchpreis 2008.

Die meisten der genannten Romane bieten ein breites Panorama des Lebens in der DDR, in dem auch alltägliche Situationen, wie das gemeinsame Essen, nicht ausgespart werden. Der vorliegende Beitrag analysiert ausgewählte Szenen gemeinsamer Nahrungsaufnahme in den Texten Kruso (2014) von Lutz Seiler, Brüder und Schwestern (2013) von Birk Meinhardt und Schneckenmühle (2013) von Jochen Schmidt 2 . Dabei soll vor allem herausgearbeitet werden, welche Funktion diese Szenen in den Texten haben. Die These ist, dass sie einerseits das Hauptthema der Texte - Leben in der DDR - aufnehmen und widerspiegeln: die Essensszenen geben Beispiele für das alltägliche Leben in der DDR, sie zeigen die Stellung der Einzelnen dazu, können Widerstand genauso wie Konformität ausdrücken. Andererseits dienen sie dazu, bestimmte, individuelle Aspekte im Text zu unterstreichen.

Die literarische Darstellung gemeinsamer Nahrungsaufnahme wird damit aus sozio-politischer Perspektive gelesen und kommt dem nach, was Dorothee Kimmich und Schamma Schahadat zum Thema Essen allgemein festhalten: „Essen erscheint als eine Art Koordinatensystem, in dem der Essende sich - je nachdem, wo er isst, was er ist [sic, hier kann wohl aber gleichzeitig ,isst“ eingesetzt werden] und wie er isst - soziologisch, politisch, wirtschaftlich etc. positioniert.“" ${ }^{3}$ Dementsprechend eignen sich Beschreibungen von Mahlzeiten, Nahrungsmitteln, dem Kochen etc. in literarischen Texten unter anderem für die Herstellung des zeitgeschichtlichen Kontextes und für soziale wie politische Kommentare. Dass solch eine sozio-politische Perspektive besonders bei literarischen Texten, die (auch) historische Ereignisse verarbeiten, ein fruchtbarer Zugang ist, hat Alois Wierlacher in seiner grundlegenden Studie Vom Essen in der deutschen Literatur gezeigt ${ }^{4}$. Dort analysiert er z.B. Thomas Manns Roman Buddenbrooks: Verfall einer Familie (1901) und zeigt, wie der soziale Abstieg der Familie nicht zuletzt in den drei größeren dargestellten Mahlzeiten widergespiegelt wird5. In Joseph Roths Radetzkymarsch (1932) hingegen repräsentieren sowohl die ritualisierten Essgewohnheiten im Hause von Trotta als auch eine Essenseinladung von Trottas durch Graf Chojnicki die militärische Macht der Habsburgermonarchie und gleichzeitig ihren

2. - Lutz, Seiler, Kruso, Berlin, Suhrkamp, 2014; Birk, Meinhardt, Brüder und Schwestern, München, Hanser, 2013; Jochen, Schmidt, Schneckenmühle, München, btb, 2014 [2013].

3. - Dorothee, Kimmich; Schamma, Schahadat: „Vorwort: Essen“, in: Zeitschrift für Kulturwissenschaften, 2012, 1, S. 7-17, S. 9.

4. - Alois, Wierlacher, Vom Essen in der deutschen Literatur. Mahlzeiten in Erzähltexten von Goethe bis Grass, Stuttgart, Verlag W. Kohlhammer, 1987.

5. - Vgl. ebd., S. 47-51. 
Verfall ${ }^{6}$. Solche Darstellungen von Mahlzeiten in der Literatur sind Sozialsituationen, bei denen der pragmatische und lebensnotwendige Faktor der Nahrungsaufnahme in den Hintergrund tritt. Das Essen in der Literatur kann in diesem Sinne mit Wierlacher in vier Typen von Mahlzeiten systematisiert werden, auf die ich mich auch in der folgenden Analyse beziehe:

1. Schauspielmahlzeiten oder Repräsentationsmahlzeiten, wie z.B. bei den Buddenbrooks, wo die Mahlzeit Repräsentationszwecken einer Gruppe, z.B. einer Familie, dient; die Speisen sind meist üppig und besonders schwer.

2. Solidarmahlzeiten, die meist in kleinen Gruppen bzw. zu zweit stattfinden. Oft lernen sich die Personen erst bei diesem Essen kennen, das somit vor allem verbindende Funktion hat. Serviert wird vorwiegend leichte, oft frugale Kost.

3. Konfliktmahlzeiten finden meist unter Erwachsenen statt, eine Ausnahme bilden Familienmahlzeiten, die oft erhebliches Konfliktpotential zwischen den Generationen bieten. Im Vordergrund steht der Konflikt zwischen den Personen. Ebenfalls zu den Konfliktmahlzeiten zählen Tribunalmahlzeiten, bei denen die Esssituation die Funktion eines Gerichts übernimmt. Die Speisen bei Konfliktmahlzeiten sind größtenteils eiweißhaltig, oft auch fett.

4. Äquivalenzmahlzeiten schließlich sind ausgeglichene Speisen, die „das diätetische Prinzip der gemischten Speisen“ berücksichtigen ${ }^{7}$.

Daneben ist für eine Systematisierung der Mahlzeiten in literarischen Texten die Zubereitungsart relevant - warme oder kalte Speisen kommunizieren jeweils andere Werte. Eine solche qualitative Reihung der Zubereitungsarten bezieht sich auf Claude Lévi-Strauss' ,Kulinarisches Dreieck' (triangle culinaire), ein Modell, in dem er die drei Zubereitungsarten Kochen, Braten und Räuchern in ihrem Verhältnis zum Rohen, Zubereiteten und Verdorbenen beschreibt. Wenngleich die Vorstellung davon, was Nahrung ist und was nicht, aber auch ,was eine jede [Gesellschaft] unter ,roh', ,gekocht' oder ,verfault' versteht" von jeweils anderen kulturellen Konventionen abhängt ${ }^{8}$, so ist der Status der Zubereitungsarten nach Lévi-Strauss universell: Das Gebratene habe gegenüber dem Gekochten stets einen höheren Prestigewert; das Kochen sei eine sparsame, plebejische Zubereitungsart, das Braten hingegen, das mit Zerstörung und Verlust einhergeht, aristokratisch ${ }^{9}$.

6. - Vgl. ebd., S. 171-176.

7. - Ebd., S. 60.

8. - Claude, Lévi-Strauss, „Das kulinarische Dreieck“, in: Helga, Gallas (Hg.), Strukturalismus als interpretatives Verfahren, Darmstadt, Luchterhand, 1972, S. 1, zitiert nach Schamma, Schahadat, „Essen: „Gut zu denken“, gut zu teilen“, in: Zeitschrift für Kulturwissenschaften, 2012, 1, S. 19-29, S. 21.

9. - Vgl. Claude, Lévi-Strauss, „Le triangle culinaire“, in: L’Arc, 1965, 26, S. 
Neben dem Servierten und der Zubereitungsart desselben sind bei einer literaturwissenschaftlichen Analyse von Mahlzeiten außerdem Aspekte wie die Personenkonstellation der Essenden, die Erzählperspektive, die Darstellung der Zubereitung sowie der fertigen Speisen, die Situation bei Tisch (Sitzordnung, Gedeck, Geschirr etc.), eventuelle Vermittlung kultureller Besonderheiten (z.B. spezifische Nahrungsmittel), um nur einige zu nennen, zu beachten.

\section{Der „Persotisch“6 als Ort der Repräsentation und der Solidarisierung in Lutz Seilers Roman Kruso}

Lutz Seilers Roman Kruso beginnt mit einem Unglück: Edgar (genannt Ed) Bendlers Freundin ist in Halle an der Saale bei einem Straßenbahnunfall gestorben, der Germanistikstudent verlässt daraufhin die Stadt, in der er sich selbstmordgefährdet wähnt. Er flieht im Frühsommer 1989 nach Hiddensee, jener Insel in der Ostsee, die zu DDR-Zeiten als Nische für Aussteiger und Andersdenkende galt. Dort beginnt Ed im Klausner als Abwäscher zu arbeiten, einer Gaststätte im nördlichsten Teil der Insel, und es entwickelt sich eine enge, aber auch schwierige Freundschaft mit seinem Kollegen Alexander Krusowitsch, genannt Kruso. Dieser ist eine Art Guru der Insel, der eine eigene Philosophie und Praxis entwickelt um die vielen Fluchtwilligen, auch Schiffbrüchigen genannten, die die DDR von Hiddensee übers Meer Richtung Dänemark verlassen wollen, mit bestimmten Ritualen zur wahren, d.h. zur inneren Freiheit zu führen und sie so vor dem sicheren Tod zu bewahren (einer der realen Hintergründe für Seilers Roman sind die Spuren der Menschen, die bei ihrer Flucht über die Ostsee verschollen sind).

Auf den allerletzten Seiten des Buches Kruso, nach dem ausführlichen Epilog am Ende des Romans, stoßen die LeserInnen auf eine Skizze (478-479), die sie zurückbringt an den Beginn des Textes. Es handelt sich um eine Zeichnung des sogenannten „Persotisches“, jenes Tisches im Klausner, an dem das Personal sich regelmäßig zu gemeinsamen Essen zusammensetzt. Seiler selbst bezeichnet diesen Tisch als den zentralen Ort, den er im Schauplatz, dem Klausner, schaffen wollte ${ }^{10}$.

19-29, S. 23. Vergleiche dazu außerdem Pierre Bourdieu, der Kochen ebenfalls als mindere Zubereitungsart bezeichnet, allerdings weil sie viel Zeit beansprucht und deshalb, entsprechend der traditionellen, spezifischen Art der Arbeitsteilung zwischen den Geschlechtern aus, eine meist den Frauen zugewiesene Zubereitungsart sei. Vgl. Pierre, Bourdieu, Die feinen Unterschiede. Kritik der gesellschaftlichen Urteilskraft, Frankfurt/ Main, Suhrkamp Verlag, 1998 [1979], S. 304.

10. - Vgl. „Lutz Seiler über die Figuren in seinem Roman Kruso“, http://www. suhrkamp.de/lutz-seiler/kruso_1206.html [8.10.2015]. 


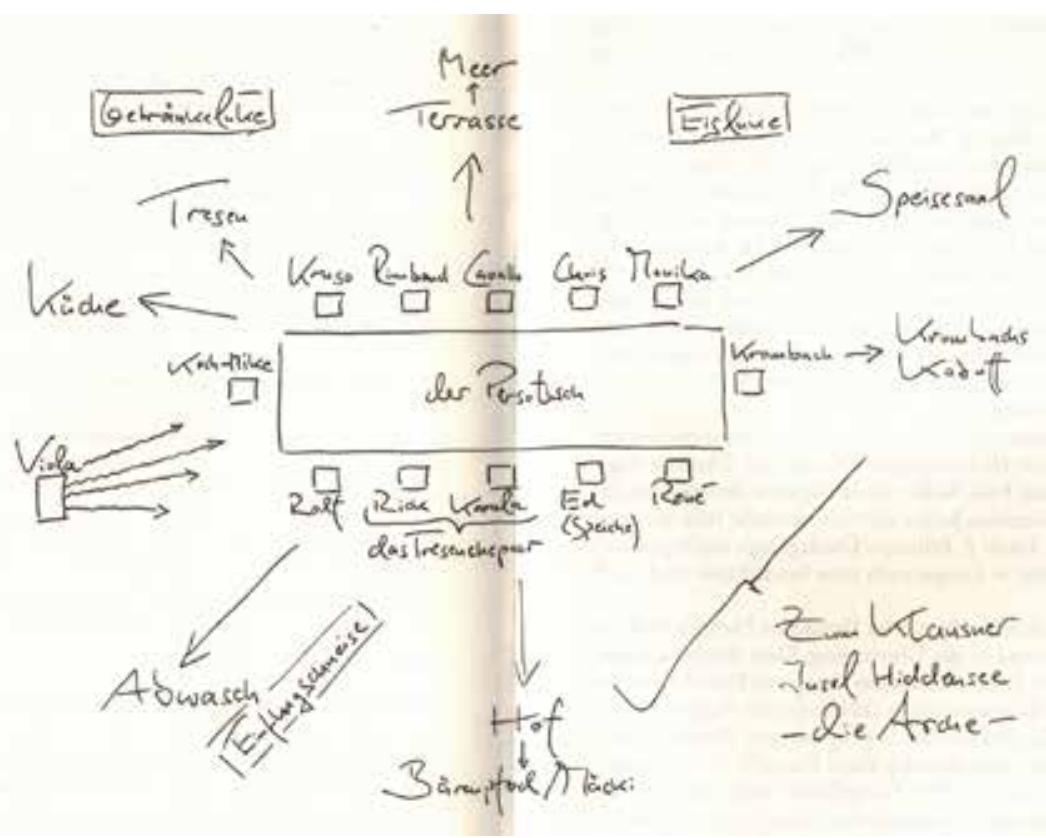

Skizze des Persotisches aus Lutz Seilers Roman Kruso (S. 478-479)

Die Mahlzeit, die auf diese Weise tatsächlich mehr oder weniger ungestört und gemeinsam eingenommen werden kann, ist das Frühstück; zu dieser Tageszeit sind die Tagestouristen noch nicht auf der Insel bzw. noch nicht bis an deren nördlichsten Zipfel vorgedrungen und die Mitarbeiter des Klausners können sich gemeinsam auf den Tag vorbereiten. Einem solchen Frühstück ist ein ganzes Kapitel (betitelt mit „Das Frühstück“) gleich im ersten Viertel des umfangreichen Romans gewidmet. Es dient der genaueren Vorstellung sowohl des Ortes - des Klausners - als auch der Protagonisten, also des Personals der Gaststätte. Gleichzeitig werden hier Grundmotive des Romans eingeführt bzw. manifest gemacht, wie die Erhöhung des Alltäglichen, die sich durch die spezielle, entlegene Position des Klausners sowie die Individualität seiner ,Besatzungsmitglieder und ihrer besonderen, wichtigen Aufgabe, d.h. der Aufnahme von ,Schiffbrüchigen', ergibt, das Motiv des Schiffes, mit dem der Klausner verglichen wird sowie der zeithistorische Kontext der DDR.

Eine Einordnung der Mahlzeit in Wierlachers Typologie ist nur ungenau möglich: Sie entspricht einerseits einer Solidarmahlzeit, weil sie in einer zwar nicht zu kleinen, aber doch klar definierten Gruppe stattfindet und weil sie stark verbindende Funktion hat - die Mitarbeiter des Klausners werden (tagtäglich) auf einen neuen, gemeinsam zu bestreitenden Tag eingeschworen. Die typischen Nahrungsmittel eines 
Frühstücks - ,[e]s gab Brötchen, Brot, Leberwurst, Teewurst, ein paar Schmelzkäseecken, etwas Salami, Schnittkäse und einen zähen, zittrigen Block Mehrfruchtmarmelade auf einem Teller" (83) entsprechen der meist leichten, frugalen Kost, die Wierlacher als typisch für eine Solidarmahlzeit bezeichnet. Andererseits ist das bestimmte Frühstück, das im 13. der insgesamt 64 unnummerierten Kapitel im Mittelpunkt steht, eine Repräsentationsmahlzeit: Es dient dazu, die Belegschaft des Klausners vorzustellen, die sich gleichzeitig in ihrer Besonderheit repräsentiert - gegenüber Ed, aber auch gegenüber den LeserInnen. Verstärkt wird dieser Eindruck durch den theatralen Charakter der Szene. So entspricht das Erscheinen des Direktors des Klausners, Werner Krombach, einem Theaterauftritt: „Nachdem alle Platz genommen hatten, öffnete sich der Verschlag [d.i. die Tür zu Krombachs kleinem Büro] und Krombach trat hinter seinen Stuhl“" (82). Auch die gleich zu beschreibenden Anklänge an einen christlichen Segensspruch verstärken den theatralen Eindruck. Näher präsentiert wird bei der Mahlzeit gleichzeitig der Ort der Handlung sowie die oben angesprochenen Grundmotive des Romans. Allerdings ist ein Frühstück keine typische Speise für Repräsentationsmahlzeiten: das Servierte ist weder üppig noch schwer, auch ist die Tages- und Uhrzeit - man frühstückt im Klausner um punkt sieben Uhr (80) - für Repräsentationszwecke eher wenig geeignet.

Das erste Frühstück, an dem Ed teilnimmt, wird genau datiert, es findet am 21. Juni 1989 statt, einige Tage nachdem er begonnen hat, im Klausner zu arbeiten (80). Die Szene wird zu Eds Initiation im Klausner, seiner „Aufnahme“ (80, Hervorhebung im Text), wie es im Buch bezeichnet wird, die am Ende des Frühstücks erfolgreich abgeschlossen ist: „Ed gehörte zur Besatzung“ (88) lautet der letzte Satz des Kapitels. Gleich zu Beginn wird die Szene beschrieben: „Jeden Morgen um sieben Uhr war die Tafel komplett eingedeckt. Zwölf Teller, je fünf an den Längsseiten, zwei an den Stirnseiten.“ (80) Die „Tafel“ - und hier wird das deutlich, was ich oben als „Erhöhung des Alltäglichen“ bezeichnet habe - weckt Assoziationen mit der Tafelrunde (wenngleich der Persotisch freilich nicht rund wie jener Artus', sondern rechteckig ist), mit Eigenschaften wie „Ritterlichkeit, Ehre, Stolz“ und die Idee einer ,eingeschworenen Gemeinschaft, die auf ewig zusammenhalten soll“11, wie es Seiler selbst beschreibt. Bei John Dryden zählt die Tafelrunde des König Artus ebenso wie der Persotisch zwölf Personen, d.h. zwölf Ritter (in anderen Versionen sind es deutlich mehr) ${ }^{12}$, die Zahl zwölf lässt darüber hinaus an die zwölf Apostel denken. Diese Assoziation wird zudem nahegelegt durch „eine winzige Reproduktion

11. - Ebd.

12. - Vgl. John, Dryden, „King Arthur“, 1691. 
des Abendmahls von Leonardo“ (86), die Ed während des Frühstücks an einer Wand zwischen Fotos der Belegschaften vergangener Jahre entdeckt. Zudem erhält die gesamte Frühstücksszene eine christliche Konnotation durch die Assoziationen, die Ed mit den Handbewegungen Werner Krombachs, dem Direktor des Klausners, bei seiner Vorstellung der Mitarbeiter verbindet: „Das Auf und Ab seiner weichen, auf Kopfhöhe erhobenen Hand und ihr Weiterrücken im Halbkreis von Stuhl zu Stuhl erinnerte Ed an die Erteilung des Segens.“ (85) Es ist nicht nur der Segen eines gewöhnlichen Priesters, sondern erinnert an jenen urbi et orbi-Segen des Papstes, wenn der Direktor seine Hände hebt „als wollte er den Erdkreis einbeziehen in seinen Segen“ (85). Schließlich hält er, zum Abschluss der Vorstellungsrunde, in der er auch Ed ,mit einer halb erfundenen und halb zutreffenden Beschreibung [...] präsentierte“ (84), dem Neuankömmling seine „Segenshand“ (87) entgegen. Bereits in den vorangegangen Kapiteln wurde Kruso, der auch am Frühstückstisch sitzt, nicht zuletzt als jemand präsentiert, der sich um die sogenannte ,heilige Suppe“ (72, Hervorhebung im Text) für die Pilger kümmert - er ist damit ebenfalls christlich-religiös konnotiert. Sowohl die Tafelrunde als auch die christlichen Assoziationen unterstreichen die eingeschworene Gemeinschaft, die sich hier zum gemeinsamen Mahl trifft und machen das beschriebene Frühstück zu einem wahren Initiationsritual.

Gleich zu Beginn des Kapitels wird die Charakterisierung des Klausners als Schiff unterstrichen. Es ist die „Besatzung des Klausners“ (80), die sich zum Frühstück trifft. Diese Metaphorik zieht sich durch das ganze Kapitel (und durch den gesamten Roman): Als Krombach sich zum Tisch setzt, murmelt er ,ein paar belanglose Sätze über das Wetter in der Nacht, die Strömung, den Wellengang und den Wind am Morgen, als ginge es darum, zum Fischfang auszufahren.“ (82f) Als er schließlich seine Vorstellungsrunde beginnt, lenkt er die Aufmerksamkeit der MitarbeiterInnen mit ,Matrosen, ich möchte euch Edgar Bendler vorstellen." (84, meine Hervorhebung) auf sich; der Klausner wird von ihm als „unsere Arche“ (87) bezeichnet. Die Geräuschkulisse, vornehmlich das „Schrappen der Ventilatoren“ (87) an der Decke versetzt die Gaststätte in Eds Wahrnehmung in eine andere Gegend, die auch „,irgendwo im offenen Meer“ (87) liegen könnte. Für ihn spricht Krombach „wie ein wirklicher Kapitän“ (88). Die Szene endet schließlich mit dem Ruf „All hands on deck!“ (88), also dem obligaten Seemannsruf „Alle Mann an Deck!“, zu dem alle ihre Kaffeetassen erheben. - Wieder einmal muss die Besatzung des Klausners das große Schiff durch die stürmischsten Stunden des Tages (die Mittagszeit, wenn alle Ausflügler gleichzeitig ihr Mittagessen verlangen) bringen.

Der zeithistorische Kontext schließlich, d.h. das Leben in der DDR, wird bei dem Frühstück durch kleine Hinweise hergestellt. So erwähnt 
Krombach seine gemeinsame Vergangenheit mit dem sogenannten „Tresenehepaar“ (85) Karo und Rick, einem tatsächlichen Paar, mit dem ihn ,eine Hauptstadt-Vergangenheit, nicht wahr, sagen wir eine Palastgeschichte“ (85) verbindet. Hier wird auf den FriedrichstadtPalast angespielt, eine große Veranstaltungsstätte in Berlin, die dank der Ausstrahlung der beliebten Fernsehsendung Ein Kessel Buntes in der ganzen DDR bekannt war und in der Krombach sowie Karo und Rick beschäftigt waren. Schließlich spricht Krombach die Situation auf der Insel bzw. deren Bedeutung an, denn sie war ,unserer Rettung [...], als uns nicht etwa das Meer oder ein Fisch, sondern das Land ausgespuckt hat" (86). Die, die es im Land nicht mehr aushalten, die fliehen wollen, aber doch die DDR nicht verlassen dürfen, versuchen auf diese Weise zumindest an ihre äußerste Grenze zu gelangen und fühlen sich, wie Ed, weit ,von Land und Staat entfernt“ (87): Künstlern, Schauspielern, Schriftstellern, Musikern, aber auch Wissenschaftlern wird Hiddensee zu DDR-Zeiten ein Treffpunkt. Freilich weiß der Staat um diese Situation und die Bewachung funktioniert auch auf Hiddensee; allerdings gestattet man den ,Aussteigern" auf dem überschau- und kontrollierbaren Eiland relative Freiheit. Die im Roman als Schiffbrüchigen bezeichneten schließlich sehnen sich nach der Freiheit, die von Hiddensee aus in der Form der dänischen Insel Møn bei gutem Wetter sichtbar ist. Viele wagen einen Fluchtversuch; Kruso sieht es hingegen als seine Aufgabe, die Menschen von diesem meist tödlichen Unterfangen abzubringen. Die Aufarbeitung der Geschichte der Geflüchteten findet sich in Eds Epilog im Roman. Dem Autor Lutz Seiler ist es bei seinen Recherchen, u.a. in Kopenhagen, gelungen, die Identität von 15 der insgesamt 174 Todesopfer herauszufinden und weiteres Licht in ein Kapitel der DDR-Geschichte zu bringen.

Ein scherzhafter Hinweis auf den historisch-kulturellen Kontext schließlich ist eines der Pflegeprodukte, die Krombach verwendet. Er ist stets ,gehüllt in eine Wolke Exlepäng“ (82), ein in der DDR vertriebenes Haarwuchsmittel.

\section{Ein thüringischer Leichenschmaus in Birk Meinhardts Brïder und Schwestern}

Birk Meinhardts umfangreicher Roman Brüder und Schwestern erzählt die Geschichte der Familie Werchow in den Jahre 1973 bis 1989. Im Mittelpunkt stehen dabei Willy Werchow und seine Frau Ruth sowie ihre drei Kinder Britta, Erik und Matti, die in der fiktiven thüringischen Stadt Gerberstedt an der (ebenfalls fiktiven) Schorba leben. Willy ist Direktor der lokalen großen SED-eigenen Druckerei „Aufbruch“ und ein entsprechend einflussreicher Mann. Die Geschichte der Familie bzw. ihrer einzelnen Mitglieder wird in enger Verflechtung mit dem 
zeithistorischen Kontext der DDR erzählt. Das trifft sowohl auf Willy $\mathrm{zu}$, aber auch auf Britta, die als junge Frau eine Karriere im Zirkus beginnt, auf ihren Bruder Matti, der als Flussschiffer einen alternativen DDR-Alltag erlebt und an Bord einen Roman schreibt, der im Westen erscheint, und schließlich auch auf Erik, der Karriere im Außenhandel im sogenannten befreundeten Ausland macht.

Das Buch fängt mit einer zeitlich vor der eigentlichen Handlung angesetzten Episode an, die der jugendliche Willy und sein Freund, Achim Felgentreu, im Thüringen der Nachkriegszeit erleben. Das zweite Kapitel mit dem Titel „Die Trauerfeier“, setzt 1973 ein und beginnt mit Rudolf Werchows, Willys Vater, Beerdigung. In diesem Kapitel findet sich die zentrale Essensszene, die im Folgenden analysiert werden soll: nach dem Begräbnis lädt Willy „alle noch zum Leichenschmaus ein“ (29). Dieser Teil der Trauerfeier dient, wie schon das Begräbnis davor, zur Vorstellung und weiteren Charakterisierung der wichtigsten Figuren des Romans, das Essen bietet dafür den geeigneten Rahmen: Die Protagonisten sind alle an einem Tisch versammelt, Gespräche können stattfinden, Blicke gewechselt werden, der allwissende Erzähler kann gleichzeitig auf die Gedanken und Erinnerungen der einzelnen eingehen. Die Szene bietet die Möglichkeit, auf Ereignisse der Vergangenheit zu verweisen bzw. diese zu erzählen und zugleich die Verhältnisse in der Gegenwart darzustellen. Auch die Entwicklung der Beziehungen zwischen den handelnden Personen kann auf diese Weise gezeigt werden. Zudem wird der politische und zeitgeschichtliche Kontext beim Essen deutlich - die Trauerrede beim Begräbnis, in der es unter anderem um die politische Vergangenheit Rudolf Werchows ging, bildet dafür die Basis. Ost und West sind durch die Anwesenden vertreten, in ihrer stärksten Ausprägung in Form von Herbert Rabe, ,langgedienter Erster Gebietssekretär der Sozialistischen Einheitspartei Deutschlands“" (21) und, andererseits, Bernhard Werchow, Willys Bruder, der mit seiner Familie in Bayern lebt und mit seinem BMW zum Begräbnis seines Vaters kommt, allerdings zu spät - aufgrund der langen Grenzkontrolle versäumt er die Beerdigung und stößt erst beim Leichenschmaus zur Gesellschaft.

Willy Werchows bereits zitierte Einladung zum Leichenschmaus charakterisiert die Mahlzeit eigentlich als Solidarmahlzeit. Allerdings ist den Anwesenden sofort klar, dass es sich um eine symbolische Einladung handelt, wie auch Willy Werchow selbst denkt (29): erschrocken schütteln „Wanderfreunde und alte Nachbarn“ (29) die Köpfe und verabschieden sich schnell. Übrig bleibt die Familie Werchow sowie deren engste Freunde, Achim Felgentreu mit seiner blinden Mutter Clara und seinem Sohn, Jonas, Ruths beste Freundin Marieluise Wehle und ihre Tochter Catherine. In dieser Besetzung hätte die Mahlzeit zwischen Repräsentationsmahlzeit (die ein Leichenschmaus zwangsläufig auch 
immer ist) und Solidarmahlzeit angesiedelt sein können. Allerdings haben nicht alle den symbolischen Wert derEinladung anerkannt: Rudolfs letzter Nachbar, Heiner Jagielka, der Gärtner der Stadt, taucht in seinen für den Anlass unpassenden - Arbeitskleidern auf, neben ihm erscheint der bereits erwähnte Herbert Rabe. Vor allem mit letzterem am Tisch ist eine Solidarmahlzeit nicht möglich, da eine Solidarisierung von Seiten der Familienmitglieder und der Freunde der Familie nicht erwünscht ist. Wenngleich Willy selbst Parteimitglied ist, empfindet er Rabe als Eindringling beim Leichenschmaus, was vor allem damit zu tun hat, dass Rabe Willys Vater aus der SED-Gebietsleitung verdrängt hat, wie die LeserInnen im Laufe des Begräbnisses erfahren haben (vgl. 24). Die Mahlzeit wird durch diese unvermittelte Wendung gleich zu Beginn zur Konfliktmahlzeit; einige Kennzeichen einer Repräsentationsmahlzeit, bei der in diesem Fall die (erweiterte) Familie repräsentiert wird, bleiben allerdings erhalten und charakterisieren den Leichenschmaus. Dies sind vor allem die anwesenden Familienmitglieder und Freunde und der Ort, das lokale Gasthaus „Sonne“ in Gerberstedt, sowie die servierten Speisen, die eine Mischung aus lokaler und überregionaler Hausmannskost darstellen. Diesen Aspekten wohnt aber gleichzeitig wiederum Konfliktpotential inne: Eine Familienmahlzeit ist mit Wierlacher immer auch eine potentielle Konfliktmahlzeit. Oft wird die Auseinandersetzung in Form eines Generationenkonflikts deutlich, im vorliegenden Fall geht es vor allem um die (durch Herbert Rabe provozierte) Konfrontation zwischen den Brüdern Willy und Bernhard.

Die Speisen, die - schwer, fettig bzw. eiweißhaltig - ebenfalls sowohl auf eine Repräsentations- als auch eine Konfliktmahlzeit verweisen, sorgen auch für Missstimmung, zumindest zu Beginn des Essens. So wird das Scheitern der intendierten Repräsentationsmahlzeit am Anfang deutlich und manifestiert sich im Laufe der Szene. Für eine Repräsentationsmahlzeit spricht, dass Willy „mit dem Chef der ,Sonne““ (30) die Speisen des dreigängigen Menüs vereinbart hat - bei jedem Gang stehen zwei Optionen zur Wahl: „Eierflockensuppe oder Soljanka, Schweinebraten mit Thüringer Klößen oder Schnitzel mit Kartoffeln, Birnen- oder Kirschkompott" (30). Die Abmachung über die Speisenauswahl funktioniert aber gleich zu Beginn des Essens nicht: Die Kellnerin serviert ausschließlich Eierflockensuppe, die sie den Ersten ohne zu fragen „hinknallt“ (30), wobei die Flüssigkeit auf die Untertassen schwappt. Erst nach einem kurzen Streitgespräch zwischen Willy und der Kellnerin, ihrem kurzen Verschwinden und einer Intervention Willys, wohl bei der Leitung des Gasthauses,

erschien die Kellnerin mit einem Tablett, darauf Eierflockensuppe und Soljanka, und fragte mit devoter Verbeugung, schräg gehaltenem Kopf, vorgestelltem Bein und breitgezogenem Lächeln, kurz, mit einer 
so übertriebenen Liebenswürdigkeit, dass alle das schon wieder als Frechheit verstehen mussten: ,Dürfte ich wohl bitte erfahren, wer von den Herrschaften unsere Eierflockensuppe wünscht und wer unsere Soljanka?‘ (31)

So wird über das Essen und dessen Ordnung Macht ausgedrückt - die Macht des lokal bedeutsamen Direktors Willy Werchow, der auf höherer Ebene interveniert, aber gleichzeitig auch die Macht der Kellnerin, die die Speisen serviert und damit die (zwar vorbestimmte, aber doch gewisse Freiheiten lassende) Choreographie ausführt. Steht die Auswahl der Speisen zu Beginn noch im Mittelpunkt, vor allem, weil sie nicht so funktioniert, wie geplant, so ist sie im weiteren Verlauf des Essens kein Thema mehr. Beim Hauptgericht scheinen sich alle für den Schweinebraten entschieden zu haben, der vorzüglich schmeckt. Beim Dessert schließlich, das serviert wird, nachdem Bernhard das Lokal wutentbrannt nach einem sehr kurzen Aufenthalt wieder Richtung Bayern verlassen hat, ist die Wahlmöglichkeit in den Hintergrund gerückt. Nach dem Zwist wähnt sich die Kellnerin wieder in der stärkeren Position, sie serviert ,mit höhnischem Gesichtsausdruck“ (49) das Kompott und sagt sich ,euer Streit ist mein Vergnügen“ (49).

Die einzelnen Gänge des Essens strukturieren die Szene. Die Suppe bildet den Rahmen für den ersten Abschnitt, in der der Rhythmus noch fehlt: Die Gäste nehmen ihre Sitzplätze ein, Rabes und Jagielkas Anwesenheit sorgen für Überraschung, Unsicherheit und Ärger, die Kellnerin wird als gelangweilt bis aufmüpfig und frech beschrieben, die Wahl zwischen den beiden Suppen funktioniert nicht. Mit dem Hauptgericht (dem Braten) beginnt wortwörtlich der nächste Abschnitt: „Sie waren jetzt beim Hauptgericht.“ (31) Bei der vorzüglichen Speise entspannt sich die Situation zunächst, man findet in Gespräche, ein Rhythmus wird erkennbar. Er wird unter anderem durch die Bewegungen der Kellnerin evoziert: Sie „bediente nun endlich so, wie es angeraten war, unauffällig und aufmerksam, nicht zu hastig, nicht zu langsam.“ (31) Die Fleischspeise und ihre Beilagen werden genau (und mit großer Lust sowie etwas Ironie) beschrieben:

Das charakteristisch Thüringische drückte sich aus in der würzigen, den Gaumen buchstäblich kitzelnden Kruste des Bratens; in der riffligen Glasigkeit der Klöße; in den fettgetränkten und gleichwohl rauchigen Bröseln; in dem bissfesten, zwischen den Zähnen zu Creme zerlaufenden Rotkohl; und schließlich in der wohligen Schwere einer Soße, die ihre Grundsubstanz, ordinäres 405er Mehl, perfekt zu verbergen wusste. (31)

Der Braten stellt für Bernhard, den Bruder Willys, der seine Familie seit vier oder fünf Jahren (41) nicht mehr gesehen hat, da er 
in Bayern lebt, den „Geschmack seiner Kindheit“ (41) dar. Doch der Braten bildet auch den Hintergrund zu dem Konflikt, der sich zwischen Rabe und Bernhard, und schließlich auch Willy, entzündet. Mit LévyStrauss wohnt der gewählten Speise die Auseinandersetzung zwischen den Männern bereits inne: Braten ist zwar ein Kochvorgang, der der Natur näher steht als das Kochen, für das ein Behältnis notwendig ist. Allerdings wird der direkte Kontakt der Nahrung mit dem Feuer in vielen Kulturen mit dem Männlichen assoziiert, genauso wie generell Fleischspeisen, und hat Konnotationen wie Zerstörung und Verlust, da Teile der Nahrung verloren gehen oder verbrennen können.

Das bereits beschriebene Servieren des Desserts schließlich leitet den letzten Abschnitt des zweiten Kapitels ein.

Neben den Speisen strukturiert aber auch das Essen (als Tätigkeit, als Verb) den Text bzw. sorgt es für den Rhythmus der Szene: Das Kauen, Schneiden, Essgeräusche, Gesten, aber auch Besteckklappern und Gläserklirren, Gerïche etc. sind oft eng mit dem Gesagten und Erzählten verbunden. So begleitet der stets abnehmende Dampf aus den Tellern der Eierflockensuppe das Warten auf die Kellnerin in der ersten Szene des Essens: „Schweigend sah man auf die drei erkaltenden Eierflockensuppen, unruhig verfolgte man, wie der letzte matte Dampf aus den Tassen stieg." (30) Und der vorzügliche Geschmack des Bratens sowie die Geräusche des Bestecks und der Gläser erleichtern es, in Gespräche zu finden: „Zunächst flogen nur vereinzelte, wie verschämte Worte hin und her, dann wurden es mehr, und das Besteck klapperte auch lauter, Gläser klirrten aneinander, alles schien in einer großen Erleichterung zu münden.“ (31) Clara Felgentreu, die blinde Mutter Achims, muss sich das Fleisch von ihrem Sohn zerkleinern lassen, erkennt aber am Schleifgeräusch des von ihrem Sohn herübergeschobenen Tellers, wann sie zu essen beginnen kann (34). Während des Essens erzählt sie von ihrem Mann und einem Gummiknüppel, mit dem dieser einst Herbert Rabe verdroschen hat; dabei umklammern ,ihre zittrigen Fäuste [...] Messer und Gabel, die wie windgeschüttelte Standarten nach oben ragten.“ (35) - Das Besteck wird für Clara Felgentreu zur Fahne, die ihren Mann und ihre Familie symbolisieren und die sie trotz ihres Alters und ihrer Blindheit möglichst aufrecht hält. Bernhard schließlich schiebt ,seinen Teller so ungestüm beiseite, dass sich das Tischtuch zu einem kleinen Faltengebirge formte" (44), als er sich von Herbert Rabe bedroht wähnt. Nachdem am Ende der Mahlzeit Jonas Felgentreu ein höchst poetisches, verschlüsseltes, aber zumindest anscheinend systemkritisches und gegen Rabe gerichtetes Gedicht rezitiert, herrscht Stille im Seitengewölbe des großen Saals der „Sonne“. Das leise Besteckgeklirr, das aus dem vorderen Teil der Gaststätte zu hören ist, ,klang wie aus einer anderen, harmonischeren Welt“ (52). Die Geräusche am Tisch hingegen haben keinen angenehmen Beigeschmack, 
sie kommen von Herbert Rabe: „Er lutschte hörbar an dem kleinen Löffel, den er aus seinem schon geleerten Kompottschälchen genommen hatte. Dann klackerte er, gesenkten Kopfes und scheinbar gedankenverloren, mit dem Löffel an das Schälchen.“ (53) Dieses Klirren macht die ohnehin schon angespannte Gesellschaft noch aufmerksamer für das, was Rabe zu Jonas sagen wird: Sein ,Urteil' über den Jungen („,Das werde ich mir bestimmt merken, junger Mann, das ist versprochen.“, 53) wird angekündigt wie in anderen Situationen ein Trinkspruch oder eine Tischrede.

\section{Essen in Ost und West in Jochen Schmidts Schneckenmühle}

Jochen Schmidts Schneckenmühle spielt wie Seilers Kruso im Sommer 1989; erzählt wird nicht nur der letzte Sommer vor der Wende, sondern auch Jens' letzter Aufenthalt im sächsischen Ferienlager Schneckenmühle - im nächsten Jahr wird der Vierzehnjährige bereits zu alt dafür sein. Der Roman erzählt in 33 Kapiteln, die jeweils in kurze Absätze gegliedert sind, aus der Sicht des Jugendlichen (und in seiner Sprache) vom Ferienlager, aber auch von seiner Familie, von der Schule und dem Alltagsleben. Dabei wird immer wieder Bezug genommen auf die Unterschiede zwischen Osten und Westen, oft auf sehr unterhaltsame Weise, wenn zum Beispiel Jens' Onkel aus dem Westen behauptet, er würde „mit Betreten der DDR Atembeschwerden“ (15) bekommen und Jens daraus schließt: „Wenn sie bei uns leben müssten, müssten unsere Verwandten aus dem Westen sterben.“ (15)

Essen wird in Schmidts Roman nur punktuell erwähnt, es werden keine größeren Mahlzeiten dargestellt. Wenn Nahrungsmittel im Mittelpunkt stehen, dienen sie entweder dazu, die Atmosphäre in dem Ferienlager zu unterstreichen und die Kinder bzw. Jugendlichen authentisch darzustellen. Andererseits werden über die Nahrungsmittel Bezüge zur DDR hergestellt bzw. wird der politische Diskurs aufgezeigt, der auch in die Konversation und die Gedanken der Jugendlichen einfließt, wobei er oft ironisch unterlaufen wird. Diese zwei Funktionen der Darstellung von Essen sind im Text häufig miteinander verflochten. Am Ende des Buches spielen Nahrungsmittel außerdem eine wichtige Rolle, als ein Mädchen, Peggy, vom Ferienlager verschwindet und der Ich-Erzähler Jens, der als einziger weiß, wo sie sich versteckt hält, sie mit Essen versorgt. Aufgrund des Fehlens von größeren Mahlzeitenszenen ist die Anwendung von Wierlachers Typologie in diesem Fall schwierig - es kann nur eine Aussage für die generelle Rolle des Essens im Text getroffen werden. Diese schwankt, entsprechend den Hauptfunktionen der beschriebenen Nahrungsmittel und Mahlzeiten, zwischen solidarisierend (bei den gemeinsamen Essen wird die Beziehung zwischen den 
Jugendlichen weiter verstärkt, das trifft besonders auch auf Peggy und Jens am Ende des Romans zu) und repräsentierend, das allerdings gegenüber den LeserInnen in Hinblick auf den zeithistorischen Kontext, nicht als Repräsentation der Gruppe der Essenden selbst. Repräsentiert wird vielmehr die DDR, der Sozialismus im Vergleich zum Westen, Differenz zwischen den Systemen wird nicht zuletzt über Nahrungsmittel hergestellt. Oft sind die beiden Funktionen des Essens - solidarisierend und repräsentierend - im Roman miteinander verbunden.

So beschreibt der Ich-Erzähler, wie einer der Jungen mit dem Besteck spielt: „Eike spielt mit seinem Löffel ,Fahrstuhl’, das Aluminium lässt sich ja leicht verbiegen, die Zähne der Gabeln zeigen immer in alle Richtungen. Eike hat einen 90-Grad-Knick in den Griff gemacht und fährt damit senkrecht vom Teller zum Mund hoch.“ (44) Hier wird einer der üblichen Späße erzählt, zu denen es kommt, wenn mehrere Kinder miteinander essen. Die Szene gibt den Alltag im Ferienlager wieder, das Essen und das gemeinsame Spiel dabei verbinden die Kinder miteinander, die Szene hat solidarisierende Wirkung. Doch sie lässt bei Jens außerdem eine Assoziation aufkommen: „Im Westfernsehen war mal Uri Geller zu sehen, der es schaffte, Löffel zu verbiegen, wir verstanden überhaupt nicht, was daran die Kunst sein sollte.“ (44) Auf diese Weise flicht Schmidt den zeithistorischen Kontext ein: die Tatsache, dass Jens (wie auch die anderen Kinder) Westfernsehen kennt, dass es dort Dinge zu sehen gibt, die das heimische Fernsehen nicht bringt, dass aber gleichzeitig dort nicht alles so großartig ist, wie es auf den ersten Blick scheint: Besteck verbiegen gelingt den Jugendlichen schließlich auch selbst.

An anderer Stelle werden die Bezüge zur DDR auf direkte Weise über bestimmte Produkte hergestellt: So erwähnt der Ich-Erzähler die „überhaupt nicht nach Schokolade schmeckende Creck-Schokolade“ (44) und hat meist eine Packung „Pfeffis“ dabei $(171,220)$. Beides sind Süßigkeiten, die in der DDR hergestellt wurden, man könnte fast von einem Product placement sprechen, das dazu dient, Atmosphäre zu schaffen, das aber auch mit dem Wiedererkennungseffekt bei den LeserInnen spielt. ${ }^{13}$ Bestimmte Westprodukte dienen ebenfalls dazu, auf die politischen Systeme zu verweisen bzw. sogar auf die verschiedenen Formen des Sozialismus. So behauptet einer der Jungen, dass es in Ungarn „Danone-Joghurt“ (98) gibt und ein anderer ergänzt: „Und Schweppes.“ (98) Nicht alle wollen das glauben, denn „Ungarn ist doch

13. - Dies erinnert an den Film Good Bye, Lenin! (2003), in dem ebenfalls mit DDR-Marken wie Spreewaldgurken, Mocca Fix Gold und Rotkäppchen-Sekt gearbeitet wird. Der Autor Thomas Brussig sieht darin den Erfolg des Films und meint: „So war das mit den Westdeutschen, denen konnte die DDR am besten als eine Markenwelt erklärt werden.“ Thea, Dorn/ Jana, Hensel/Thomas, Brussig, Sind wir ein Volk?, Freiburg, Herder, 2015, S. 31. 
sozialistisch.“ (98) Der Ich-Erzähler wiederum denkt an den Joghurt, den er normalerweise trinkt bzw. isst - er hat ,,ja immer unterschiedliche Konsistenz“ (98) - und an dessen ,weiße Plasteverpackung, die mit den Jahren immer dünner und durchscheinender geworden ist" (98). Selbst die alternative Entsorgung, die Jens und seine Schulkameraden für den Joghurt gefunden haben (wir ,werfen ihn ins Klappfenster der Rewatex-Reinigung“, 99) ist eine Bezugnahme auf den zeithistorischen Kontext - Rewatex (stand für: Reinigt und wäscht Textilien) war ein DDR-Wäscherei- und Reinigungsbetrieb. Anderen Jugendlichen im Ferienlager ist nicht nur das unterschiedliche Warenangebot in Ungarn, sondern auch die Tatsache, dass DDR-Bürger zunehmend versuchen, über das Land auszureisen, bekannt.

An anderer Stelle werden die Nahrungsmittel, die im Intershop am Dresdner Hauptbahnhof angeboten werden, zum Versprechen einer anderen, besseren Welt:

Es duftet nach Kaffee, Schokolade, Waschmittel und Parfüm. Es gibt hier nichts, was man nicht gerne hätte. [...] Die unbegreifliche Luftschokolade. Das Waffeleis ist in Papier eingewickelt, sogar die Waffel selbst schmeckt, sie ist mit Schokolade überzogen und weicht im Mund nicht auf. (135, Hervorhebung im Original)

Wiederum wird das Westproduktfür den Ich-Erzähler Ausgangspunkt für einen Vergleich mit dem ihm bekannten DDR-Gegenstück: „Bei unseren Waffeln zermantscht man immer den Rand mit seiner Spucke.“ (135) Diese Vergleiche und Verweise funktionieren in Schmidts Roman unter anderem deshalb, weil sie nur punktuell eingesetzt werden und kurz gehalten sind. Die teils naiv wirkende, direkte Sicht des jugendlichen Ich-Erzählers sorgt zudem für ironische Distanz bei den LeserInnen.

\section{Zum Abschluss}

In diesem Beitrag habe ich ausgewählte Szenen gemeinsamer Nahrungsaufnahme in drei Texten analysiert, die das Leben in der DDR literarisch verarbeiten. Dabei stand die Funktion dieser Szenen im Vordergrund, die ich mithilfe von Alois Wierlachers Typologie von Mahlzeiten in literarischen Texten und in Kombination mit einer formalen Analyse untersucht habe. Die beschriebenen Mahlzeiten bewegen sich zwischen Solidar- und Repräsentationsmahlzeit (in Kruso und Schneckenmühle) bzw. zwischen gescheiterter Solidar- und Repräsentationsmahlzeit und Konfliktmahlzeit (Brüder und Schwestern). Im Fokus der Analyse stand einerseits die jeweils individuelle Funktion der Szenen, andererseits die Frage danach, inwieweit sie das Thema Leben in der DDR aufnehmen und widerspiegeln. Diese beiden Funktionen sind in den beschriebenen Texten eng miteinander verwoben. 
Den untersuchten Beispielen in allen drei Romanen ist gemeinsam, dass sie keine Essen in privaten Räumen beschreiben. In Kruso und in Brüder und Schwestern spielen die Szenen in Gasthäusern (wenngleich der Persotisch bei Seiler als ein semi-privater Raum bezeichnet werden kann, da er dem Personal vorbehalten ist), in Schneckenmühle finden die Essen entweder im Ferienlager statt oder die Nahrungsmittel werden unterwegs oder in Geschäften (Intershop) verzehrt oder erwähnt. Die beschriebenen Orte der Nahrungsaufnahme sind damit öffentlich - die Öffentlichkeit in Form der Gesellschaft oder des Staates ist auf diese Weise präsenter als sie es in privaten Räumen sein könnte ${ }^{14}$. Dies wird am deutlichsten in der Konfliktmahlzeit in Brüder und Schwestern durch die Anwesenheit Herbert Rabes beim Leichenschmaus. In einem privaten Rahmen wäre es eher möglich gewesen, ihm den Zutritt zu verweigern oder die Einladung exklusiver auszusprechen. Auch die Rede Krombachs in Kruso hat semi-offiziellen Charakter; er kommt mehrmals auf die Rolle der Insel und der ,Besatzung' des Klausners im Verhältnis zum ,Festland' zu sprechen, der zeithistorische Kontext ist damit präsent. Bei Schmidt schließlich ergeben sich die Verweise sowie die Vergleiche zwischen Ost und West, die sich durch den gesamten Roman ziehen, unter anderem in den Gesprächen der Jugendlichen bei gemeinsamen Mahlzeiten in der Öffentlichkeit des Ferienlagers.

Wie zentral das Essen in den untersuchten Texten ist (und wie relevant damit seine genauere Untersuchung), wird nicht zuletzt auch in der Struktur der Romane abgebildet. Seiler unterstreicht die Bedeutung des Persotisches durch die im Buch abgedruckte Skizze desselben. Zudem spielt fast der gesamte Roman im Klausner und damit in einem Gasthof. Dies bedeutet, dass Nahrungsmittel, Kochen, Servieren und Essen durchgängig präsent sind ${ }^{15}$. Bei Meinhardt ist der Leichenschmaus Teil jenes Kapitels, in dem die Charaktere des Romans eingeführt werden und der damit die Grundlage für das restliche Buch bildet. Bei Schmidt schließlich kann eine solche Zentralität nicht festgestellt werden; durch die punktuelle, aber wiederholte Präsenz von Mahlzeiten und der Erwähnung von Nahrungsmitteln bildet das Essen allerdings ein wichtiges Hintergrundelement für den Roman.

14. - Vgl. dazu Tanja Rudtke, die festhält, dass der Besuch eines Gasthauses in der Literatur es ermöglicht, Begegnungen stattfinden zu lassen, „,ie [...] den Einzelnen mit der Gesellschaft, repräsentiert durch die Gegenwart der anderen Gäste, in besonderer Weise konfrontieren." Tanja, Rudtke, Kulinarische Lektüren. Vom Essen und Trinken in der Literatur, Bielefeld, transcript, 2014, S. 98.

15. - Stellenweise tritt eine richtiggehende Choreographie des Vor- und Zubereitens, Servierens, aber auch des Abservierens, Entsorgens und Reinigens in den Vordergrund, auf die im vorliegenden Beitrag nur verwiesen, aber nicht genau eingegangen werden kann. 